\title{
A comment on Ellwood-Lowe et al. (2020): Presentation and discussion of results should take the lead from the preregistration
}

\author{
John C. Flournoy \\ Harvard University
}

\begin{abstract}
Preregistration establishes transparent and verifiable links between hypotheses, statistical tests, and error-control of decisions. In this comment, I critique both the preregistration of, and the presentation of preregistered results from two studies investigating scarcity as a potential cause of the word gap. The primary goal is to illustrate how and why preregistration should guide the way results are framed, and how this might influence conclusions drawn.
\end{abstract}

Keywords: preregistration;error-control;false-positives.

This comment concerns version 1 of the preprint titled "What causes the word gap? Financial concerns may systematically suppress child-directed speech" (Ellwood-Lowe, Foushee, \& Srinivasan, 2021, now revised, in part, in response to this comment). In the spirit of open peer review, and because preregistration is still a fairly new practice, my comments specifically focus on aspects of the method and results relevant to preregistration. I would like to stress that it is quite commendable and even brave that the authors made the effort to register. Doing so almost certainly means that the manuscript will be held to a higher standard than if it was not preregistered. Yet it also improves the evidential value of the findings and quality of contribution to the literature. The authors' engagement in this practice helps to establish a norm that will improve the conduct of psychological science. These comments are intended to help improve the target paper and also to provide an example of how one might go about reviewing work that has been preregistered. The improvements the authors have made in light of these comments are also instructive, though I will not be re-reviewing the revision (this can be an exercise left for the reader).

First, to lay a solid foundation from which to constructively critique this manuscript, I will establish what I see as the primary functions of preregistration in the context of null hypothesis significance testing (NHST). At its heart, preregistration allows a reader to verify that the preconditions are in place to allow straightforward interpretation of $p$-values with respect to their function as a tool for making decisions about hypotheses which arises entirely from their relationship to the control of (usually false-positive) error rates. When we see a $p<.05$, we decide the null hypothesis is true because, reiterating what all of us learned in our graduate statistics course, this ensures (conditional on certain assumptions) we will mistakenly report that an effect is significant (a Type 1 error) only
$5 \%$ of the time over the long run. But over the long run of what? Well, specifically over the long run of repetitions of the study procedures and analyses. Preregistration allows set forth (and allow others to verify) exactly what those procedures and analyses are, and that the procedure is not dependent on the data (for more in depth discussion of these issues, see Flournoy et al., 2020; Srivastava, 2018).

Absent some sort of registration, the problem of the garden of forking paths (Gelman \& Loken, 2013) means we cannot verify (or even know) how many null-hypothesis significance tests might have been conducted, and thus cannot verify whether a $p$-value can be interpreted straightforwardly with respect to the long run false-positive error rate. Rubin (2017) explains why even the possibility of a forking path is problematic for Type 1 error rates:

Forking paths are problematic for the Neyman-Pearson approach because they imply that different tests will be conducted in different exact replications of the same study. For example, if a researcher transforms selfesteem scores because they are skewed in their original study, then they are making an implicit commitment to follow the same conditional rule in subsequent replications based on different samples (i.e., transform selfesteem scores when they are skewed but not when they are not skewed). In the context of each specific sample, this is a reasonable and justifiable rule to follow. Nonetheless, in the context of a long run of exact replications based on different samples, this conditional rule results in two different tests: one based on transformed scores and the other based on nontransformed scores. Hence, this conditional rule represents a forking path in the analysis protocol because it leads to different tests in different replications of the same study. (p. 322)

The key to obtaining a valid $p$-value in a NHST framework is to ensure that the number of possible forks is known (constraining analyses to a single path being the simplest case) and that the per-test $\alpha$-level for significance is adjusted to account for the forks. This allows, for example, data-dependent transformation rules or multiple tests of a hypothesis so long as they are all accounted for in a way that maintains the falsepositive error rate at the nominal level. Without such accountability, it is impossible to know what the obtained $p$-value indicates about the control of the long-run error rate; in turn, 
it is impossible to know how well-justified is the decision to reject the null hypothesis.

A related function of preregistration is to tie statistical tests to rhetorical hypotheses. In order to establish the hypothesisrelevant false-positive error rate, it is necessary to establish, prior to analyses, how particular statistical results lead to decisions regarding particular hypotheses. For example, one might have a single hypothesis that is appropriately tested by five different statistical tests, any one of which by itself would lead to a decision to reject the null and conclude in favor of the hypothesis. The probability of making a falsepositive statement in any one of the tests is the inverse of the probability of not making such a mistake five times in a row, or $1-.95^{5}=.23$. In such a case, one should correct for this magnification of risk across the whole family of tests in order to maintain the nominal false-positive rate, e.g., by using a $p$-value threshold of $1-.95^{\frac{1}{5}}=.01$ for each test. Without specifying which tests pertain to which hypothesis, it is impossible to know how to maintain the Type 1 error rate.

Now that these basic functions of preregistration have been outlined, I will make a few suggestions regarding how they are applied in the the authors' manuscript. For Study 1, the preregistration and reported results are generally consistent with the above principles, and the primary critique is with how the discussion treats the evidence from preregistered analyses versus unregistered analyses. The critique of Study 2 regards the large number of tests described in the preregistration, and the unclear links between the hypothesis and this family of tests. Finally, I will discuss the impact of these critiques on the manuscript's General Discussion. For the sake of brevity, I treat the preregistered primary analyses as the focal tests of the hypotheses because the secondary analyses are often described less completely.

\section{Study 1}

There are many strengths of the preregistration (http:// a spredicted.org/blind.php? $\mathrm{x}=79 \mathrm{ep} 26$ ) and its presentation in the manuscript for Study 1. It is very clear about the design of the study; the outcomes of interest (i.e., quantity and quality of child directed speech); the primary analyses (two-sample $t$-test between the scarcity-induction and control group for each outcome); and the hypothesis that is being tested: “... having parents engage with their own experiences of scarcity will systematically reduce how much they talk to their child (child-directed speech, CDS) in a subsequent play session." Moreover, the results from these tests are presented with prominence at the beginning of the results section and are clearly labeled as outcomes from the primary preregistered analyses:

For our primary pre-registered analyses, we found that on average, caregivers in the Control condition used 13 more word types, $\mathrm{t}(80.49)=-1.44, \mathrm{p}=0.076, \mathrm{~d}=-0.32$, $95 \%$ CIs of $\mathrm{d}[-0.75,0.12]$, and 44 more word tokens, $\mathrm{t}(79.39)=-1.2, \mathrm{p}=0.116, \mathrm{~d}=-0.26[-0.70,0.17]$ than those in the Scarcity condition, though this did not reach statistical significance. (p. 3)

I note one small issue here, though it would not have much effect in this instance: because multiple tests are relevant the the hypothesis, it would have been helpful for the preregistration to have discussed whether any adjustments to the $p$ values would be made and if not, why. Even though this is not prespecified, Bonferroni, or some other common adjustment would still be appropriate given that such adjustments are considered standard operating procedures in the field. Some discussion of this same issue regarding the preregistered secondary analyses would also have been warranted.

Despite the clarity of these preregistered results, the discussion seems to favor results from the unregistered exploratory analysis of a subset of caregivers who reflected on financial scarcity. It opens: "This study presents preliminary evidence that caregivers speak less to their children when they have been reminded of their recent experiences of resource scarcity," and then elaborates that

"[o]ur strongest result comes from an exploratory analysis suggesting that caregivers who reflected on having scarce financial resources spoke significantly less to their children than did caregivers who reflected on other kinds of scarcity and than caregivers who did not reflect on scarcity at all" (p. 4).

The evidential value of those analyses are extremely tentative for reasons outlined in the introduction. Given that the value of a preregistration is to allow us to verify what counts as confirmatory evidence versus exploratory hypothesis generation, it is crucial to let this distinction guide the way results are discussed. It would be more appropriate to say that the most robust results (in terms of well controlled Type 1 error rates) do not support a general effect of scarcity on child directed speech. It may then be appropriate to state that exploratory analyses suggest that future research should focus more specifically on a financial scarcity manipulation, leaving stronger statements about the effect of such a manipulation to be made in the report of that future work.

\section{Study 2}

The preregistration for study 2 (http: / / aspredicted.org/ blind. php? $x=g f 7 p d 7$ ) is also generally very clearly written. The question of interest is clearly specified: do naturally occurring experiences of scarcity reduce child-directed speech? Each of the primary analyses has obvious relevance to the main research question: conversational turns, as a proxy for child-directed speech, should be negatively impacted at the end of the month when parents' available financial capital is at its lowest point, and this effect might vary as a function of the household's income level. I will outline two critiques of Study 2. First, the use of multiple corpora, along with the possibility (but not necessity) of an interaction effect leads to a large number of primary analyses, yet there is no description of which patterns of results would or would not support the hypothesis. This leads to a potential magnification of the 
Type 1 error rate. Second, the presentation of the results in the manuscript does not distinguish the primary from secondary analyses leading to more ambiguity about how these analyses control the false positive rate across all possibly relevant analyses.

There are many possible patterns of results from the family of primary analyses, and only two of those patterns have unambiguous implications for the hypothesis being tested: if all corpora show the expected effect with and without moderation by income, the hypothesis would be supported; if none show an effect, the hypothesis would be left unsupported. However, there are quite a few other possible patters of results, which I will explicate. There are two primary models: conversational turns predicted by time-of-month; and conversational turns predicted by time-of-month, income, and their interaction. The preregistration specifies that these two analyses will be done for each of three corpora, and in a model that combines them. In total, this is eight models. Left unspecified is whether a significant result from any one of these tests would be considered evidence in favor of the hypothesis. If so, an uncontrolled test-wise significance threshold of $p=.05$ would lead to a maximum false positive rate of $1-.95^{8}=.34$. Control of the family-wise error rate is not discussed in the preregistration or the manuscript, and the pattern of results presented are clearly not all in support of the hypothesis. This leaves the reader unable how unlikely a false positive conclusion would be.

Clarifying, in the preregistration, the pattern of results that would be consistent with the hypothesis could have resulted in a much more constrained set of tests and thereby controlling the Type 1 error rate and increasing power (relative to a Bonferroni correction across all possible tests). If the hypothesis should be supported primarily by a consistent main effect across the three corpora, i.e., a rejection of the null for each test, then a per-corpus $\alpha=.05$ constrains the error rate for the family of three tests. To see why this is, take the extreme example in which there is a true effect in each of two corpora, but not in the third: one makes a false-positive conclusion only when the finding for the third is in error (and if there is no true effect in any corpus, the false positive rate is possibly as low as $.05^{3}=.00013$ ). On the other hand, if the effect merely needs to be present on average across all three corpora, then only two tests of the combined corpora would be needed - one without the interaction, and one with - and only a correction across these two tests would have been needed. Leaving this question unaddressed both limited the conceptual clarity of the hypothesis (for example, why should the effect be said to exist if it is only apparent in one or two corpora), and also led to an under-constrained set of hypothesis tests.

The issue raised above regarding the large number of preregistered primary analyses is magnified by the presentation of primary, secondary and exploratory analyses without rhetorical separation. This undermines the preregistration, possibly encouraging the reader to treat the entire set as the family of tests with relevance to the hypothesis, thereby obscuring or making impossible any link to an overall Type 1 error rate. To examine this issue in detail, we can look at the analyses as proposed in the preregistration alongside the results. Again, there are two primary models: conversational turns predicted by time-of-month; and conversational turns predicted by time-of-month, income, and their interaction.

The results section begins by reporting $\chi^{2}$ statistics from only the first of these two sets of primary analyses:

... across all corpora, the rate of conversational turns was lower on average during the last week of the month than the rest of the month (see Figure 3). For the models without any covariates, this reached significance in the Bergelson corpus, $\chi^{2}(1)=7.60, p=0.006$, but not the others, Cougar: $\chi^{2}(1)=1.28, p=0.259$; Warlaumont: $\chi^{2}(1)=0.82, p=0.259$; all corpora together: $\chi^{2}(1)=3.06, p=0.080$. (p. 5)

This is followed immediately by results from two secondary analyses from models controlling for the rate of adult words, and for the rate of of child vocalizations:

Effects held or became stronger when controlling for the overall number of adult words near the child, reaching significance in two out of the three corpora.... Interestingly, however, these effects were eliminated when controlling for the overall number of child vocalizations.... (p. 5)

Several sets of exploratory analyses follow, with the second set of primary analyses (income by time of month interactions) appearing only at the end of the results section, and only for one unspecified model comparison (though the reader can probably guess):

There was a trend-level interaction of time-of-month with family income, treated as a continuous variable, $\chi^{2}(1)=3.64, p=0.056$. (p. 7)

Mixing together the reporting of these various levels of prespecifation undermines the preregistered constraint on the universe of analyses that will be undertaken, and that will be read as the focal confirmation of a hypothesis. By reporting these secondary results alongside the primary results, the manuscript effectively presents 12 tests as being of central importance to the research question. If one considers the possibility that any of these models could have included income as an interaction, there are 24 tests that may have been considered relevant in some way to supporting or rejecting the central research question. If this is truly the universe of all possible analyses, the family-wise error rate could be as high as $1-.95^{24}=.71$. In effect, the way the results are presented re-opens the forking paths the preregistration sought to close.

\section{Discussion of the General Discussion}

The discussion, as a vehicle for highlighting and contextualizing results, should be written with attention to how a preregistration delineates those results that provide the most robust 
evidence from those that provide less. In the NHST framework, the evidential value of a particular result hinges on the validity of the method of constraining the false positive error rate, and analyses that meet this standard should be emphasized. Analyses for which the relationship between the false positive error rate and the verbal hypotheses is not well constrained should be presented as generative for future research rather than as confirmatory.

Using this as an organizing principle for the present manuscript suggests that the results from the preregistered primary analyses should be foregrounded. Those are, first, the comparison of the frequency of word tokens and types between the experimental and control condition from Study 1 ; and second, the prediction of conversational turns from time-of-month, and the time-of-month by income interaction from Study 2. Such a discussion would acknowledge that the primary results from Study 1 did not demonstrate a significant effect of the scarcity manipulation on tokens and types, and that the results from Study 2 yielded a significant association between conversational turns and time of month in one of eight preregistered primary analyses. This would lead to a necessary discussion of the non-significant effects, and finally to a generative synthesis of the secondary and exploratory analyses to guide future research.

The necessity of contextualizing the null effects in such a discussion presents another challenge that is indirectly related to preregistration. Among the primary preregistered analyses, there are quite a few null results that may be enticing to interpret, perhaps by stating something like, "there is no effect of the scarcity manipulation on word tokens or types." This inference would only be warranted if one were able to show control of the long run probability of making such a negative claim erroneously (the Type 2 error rate). Ideally, this would mirror the false-positive rate, which would mean demonstrating $95 \%$ power to detect the smallest effect of consequence (or an equivalence test, Lakens, 2017). The power calculation itself depends on the valid $p$-value thresholds established via the preregistration, with trade-offs between Type 1 and Type 2 errors. A highly powered analysis would allow conclusions that accept the null hypothesis as true under the same errorcontrol logic we use to conclude against the null and in favor of our hypothesis. Without being able to show high power to detect nearly-inconsequential effects, these null results would be inconclusive (but note that preregistration and prepublication ensures they are unaffected by reporting or publication bias thereby increasing their usefullness in meta-analyses).

Overall, using this manuscript's preregistration as the frame within which to paint the discussion seems to entail a much more tentative interpretation than is currently conveyed by the manuscript. The statement that "[a]cross two studies, we present evidence that structural constraints-in particular concerns with financial resources-may play an important role in care-givers' speech patterns," does not seem warranted. Depending on the results of a power analysis, one might even come to the opposite conclusion. Of course, the discussion should also address the secondary and exploratory analyses as information that could guided future work, and indeed the analyses should exploit the richness of the data set to whatever extent the authors deem appropriate. However, it should be clear to the reader which results are confirmatory, standing on solid frequentist footing, and which are not.

\section{Conclusion}

At the beginning of this comment, I suggested a preregistered article would likely be held to a higher standard than an article lacking any registration. Those who have newly taken up the tool of preregistration may find it jarring that it increases their exposure to critique. But the fact that the target article makes this critique possible is a mark of the article's quality, much in the same way reporting test-statistics, or a detailed methodology is. This is because scientific authority arises from the verifiability of the empirical basis of its claims. By testing their hypotheses using tools that allow the reader to verify the preconditions of frequentist inference, the authors have elevated their creative research on an important topic.

By separating out the analyses that were planned independent of the data, and highlighting certain analyses as the primary tests of their hypotheses, the authors provide a reproducible methodology for interrogating an interesting theoretical claim that suggests feelings of scarcity lead to diminished language production. We can trust the inferences for these primary tests because of the hard, and still somewhat risky, work the authors did to write these things down ahead of data analysis. The descriptions of the data provided by the secondary and exploratory analyses point to promising avenues for future investigation. My goal in writing this was to suggest how to better take advantage of the preregistration by highlighting the distinction between primary and other analyses, and thereby accentuating the unique contributions of each.

I would like to end this comment with a broader view of preregistration. While this comment has focused on the benefits and requirements, as I see them, of preregistration, I would not want to leave the authors, or any other reader, with the impression that preregistration must always be a part of valid scientific inquiry, nor even that it is a panacea. It is a very narrow, very brittle tool that can be used to establish complete reporting (i.e., cherry-picking's inverse) and the independence of decisions from meaningless idiosyncrasies of data. The frequentist, NHST paradigm that is overwhelmingly popular in our discipline requires both things. Other paradigms are not free of such requirements either: one can hack a Bayes-factor and cherry-pick from machinelearned predictions. However, there are other ways of meeting these requirements and drawing robust inferences from data (see, for example, Giudice \& Gangestad, 2021; Oberauer \& Lewandowsky, 2019; Rubin, 2020). We would do well to both deeply understand how preregistration solves certain inferential problems, and also to move beyond it. 


\section{References}

10 Ellwood-Lowe, M. E., Foushee, R., \& Srinivasan, M. (2021). What causes the word gap? Financial concerns may systematically suppress child-directed speech. http://doi.org/10.31234/osf.io/byp4k

Flournoy, J. C., Vijayakumar, N., Cheng, T. W., Cosme, D., Flannery, J. E., \& Pfeifer, J. H. (2020). Improving practices and inferences in developmental cognitive neuroscience. Developmental Cognitive Neuroscience, 45, 100807. http://doi.org/10.1016/j.dcn.2020.100807

Gelman, A., \& Loken, E. (2013). The garden of forking paths: Why multiple comparisons can be a problem, even when there is no "fishing expedition" or "p-hacking" and the research hypothesis was posited ahead of time, 17. Retrieved from http://www.stat.columbia.edu/ qelman/research/unpublished/forking.pdf

Giudice, M. D., \& Gangestad, S. W. (2021). A Traveler's Guide to the Multiverse: Promises, Pitfalls, and a Framework for the Evaluation of Analytic Decisions. Advances in Methods and Practices in Psychological Science, 4(1), 251524592095492. http://doi.org/10.1177/2515245920954925

Lakens, D. (2017). Equivalence Tests: A Practical Primer for $\mathrm{t}$ Tests, Correlations, and Meta-Analyses. Social Psychological and Personality Science, 8(4), 355-362. http://doi.org/10.1177/1948550617697177

Oberauer, K., \& Lewandowsky, S. (2019). Addressing the theory crisis in psychology. Psychonomic Bulletin \& Review. http://doi.org/10.3758/s13423-019-01645-2

Rubin, M. (2017). An Evaluation of Four Solutions to the Forking Paths Problem: Adjusted Alpha, Preregistration, Sensitivity Analyses, and Abandoning the NeymanPearson Approach. Review of General Psychology, 21(4), 321-329. http://doi.org/10.1037/gpr0000135

Rubin, M. (2020). Does preregistration improve the credibility of research findings? The Quantitative Methods for Psychology, 16(4), 376-390. http://doi.org/10.20982/tqmp.16.4.p376

Srivastava, S. (2018). Sound Inference in Complicated Research: A Multi-Strategy Approach (preprint). PsyArXiv. Retrieved from https://osf.io/bwr 48 